\title{
LAS HERRAMIENTAS DIGITALES COMO RECURSOS EDUCATIVOS EN EL ÁMBITO UNIVERSITARIO
}

\author{
Crespo López Robert ${ }^{1}$, López Carvajal Julia ${ }^{2}$ \\ Robert.crespo@educacion.gob.ec ${ }^{1}$,Zobeida.lopez@educacion.gob.ec ${ }^{2}$ \\ https://orcid.org/0000-0003-2011-6951', https://orcid.org/0000-0002-3431-71592 \\ Distrito Educativo Tarqui Tenguel, Ministerio de Educación.
}

Guayaquil-Ecuador

Recibido (03/07/20), Aceptado (21/07/20)

Resumen: La cultura de aprendizaje tecnológico cada vez se ve más recurrente desde el impacto innovador hasta ser necesario para la educación todo en base a beneficio de los estudiantes, en este artículo se presenta un análisis de las herramientas educativas en el ámbito universitario y el impacto de las mismas se realiza mediante un estudio de campo, entrevistas y encuestas en un centro de educación superior. Los datos que se presentan están acordes a una realidad que viven los docentes universitarios y estudiantes, en el mismo se detalla los beneficios que las herramientas tecnológicas pueden traer a la sociedad del conocimiento.

Palabras Clave: Cultura, recursos, herramientas.

\section{DIGITAL TOOLS AS EDUCATIONAL RESOURCES IN THE UNIVERSITY ENVIRONMENT}

\begin{abstract}
The purpose of this research is to validate the application of the Ludiexpression in the teaching learning process for the first year of basic in the private education of the Quito city; this analysis lies in the contribution that recreational activities will make in cognitive and integral development of the child, through action research and field study, trying to know how teachers transmit knowledge and generate the progress of skills in children of this level. Data was collected using mixed methodology, the collaborators were 12 teachers of different levels to whom a survey was applied, in which was evidenced the importance of the game as a methodology in order to bring about knowledge in children of those ages.
\end{abstract}

Keywords: Culture, resources, tools. 


\section{I.INTRODUCCIÓN}

El desarrollo de la tecnología a nivel mundial nace de las nuevas necesidades que esta trae, las organizaciones alrededor del mundo se plantean desarrollarse al nivel que estas exigen, el sistema educativo universitario no se queda atrás ya que siendo quien forma a los nuevos profesionales debe ingresar a ser parte de la misma, es por esto que obliga a todas las partes relacionadas a reinventarse en épocas tecnológicas, a estos nuevos sistemas de aprendizaje se los llama conectivismo [1].

Se dice que esto no es una modernización de los procesos o digitalización de los documentos sino a ser parte de los avances tecnológicos que vive el mundo, así mismo anclar nuevas estrategias didácticas que vayan de la mano de las TIC'S, el enfoque principal de cada una de estas es en el beneficio que pueden causar en torno a las necesidades de cada carrera, si bien es cierto se piensa que no todas las profesiones necesitan de la tecnología, pero ese pensamiento nos obliga a cambiar ya que el confinamiento vivido en el 2020 en torno al Sars COVID-19 nos demostró que por necesidad se debe de hacer uso de la tecnología como herramienta laboral, educativa y de comunicación.

Se presenta el uso de herramientas tecnológicas en las carreras contables como una estrategia para entrar al mundo laboral, dando competencias necesarias y suficientes para que el profesional pueda ser competitivo, ya que el mundo así lo pide, buscar un enfoque ya lejos de lo tradicional que permita crear una conciencia innovadora y ser parte de un proceso globalizado. La idea de mejorar los procesos.

La aceptación de los estudiantes a la creación de esta herramienta fue de gran acogida, porque sienten la necesidad de mejorar día a día, con procesos innovadores dentro de las aulas. Se ha aprovechado al máximo las opiniones de las personas involucradas estudiantes y docentes nos encaminó a realizar una herramienta con enfoque para cambiar el estilo dentro de los centros de estudios. Estos sistemas nos permiten tener una plataforma real de cada uno de los procesos como los de una compañía, con el fin de analizarla e interpretarla para tomar las mejores decisiones, es claro que una empresa es competitiva cuando cuenta con información real, en el tiempo real, es decir al alcance de todos.

Se indica que es necesario el uso de tecnologías en el ámbito educativo para establecer una mayor competitividad a los profesionales, de la misma manera conocer el uso de las herramientas tecnológicas ayuda al desarrollo personal de los individuos y mejoran la competencia.

\section{II.DESARROLLO}

El presente trabajo se fundamenta a las necesidades de innovación tecnológica en los diferentes procesos académicos para mejorar el aprendizaje dentro de las carreras de Contaduría Pública y Auditoría, con esto se espera obtener eficiencia en sus servicios. Siendo una necesidad de los estudiantes de la carrera antes mencionada, actualizar su metodología de aprendizaje mediante lo descrito el tener un software didáctico como herramienta de enseñanza dará un valor considerable a su carrera.

El uso de herramientas tecnológicas han desarrollado un lugar importante en el ámbito educativo ante las necesidades de innovación [2] esto va de la mano con la educación formal, de esta manera se complementa un tipo de educación tradicionalista que busca más allá en tener una educación de calidad estando a la vanguardia social el hecho de mantener este tipo de herramientas se enfoca en crear en los estudiantes la pre disposición de trabajar bajo los estándares necesarios en una época tecnológica.

Se debe abordar de manera extensa los profesionales en capacitación para que tengan las herramientas necesarias para el mercado laboral que cada vez está más competitivo, la capacitación continua es uno de los temas que debe ser constante en la profesión ya que día a día se ven mejoras, financieras, tributarias y contables, mediante leyes, reglamentos o normas y esto debemos plantearlos a nuestro diario vivir. Las herramientas tecnológicas siempre deben ir de la mano de cada profesión ya que son hechas para ahorrar tiempo en los procesos y ser más eficientes en el uso de recursos de la compañía.

En el ejercicio de la profesión contable siempre debe entregarse los estados financieros de una manera estandarizada para que terceras personas puedan conocer la situación real de una organización tanto financiera como tributaria [3], y así se puedan tomar las decisiones más acertadas dentro de la misma, de igual manera la entrega de información para instituciones externas y así cumplir con obligaciones financieras.

El propósito de esta investigación nace de la mano de la evolución informática que vive el mundo, el limitado uso de herramientas tecnológicas dentro de las carreras hace que los estudiantes pierdan competitividad en el mercado laboral [4], el punto de innovar con tecnología beneficia a la Universidad puesto que en la actualidad todos los CES (Centros de Educación Superior) se encuentran en constantes evaluaciones por parte de organismos rectores, esto nos permitirá ser un centro de estudio con formación de tercer nivel para formar profesionales con avances tecnológicos de bien, útiles para la sociedad entera y que puedan hacer valer sus 
conocimientos en las diferentes áreas donde se desarrolle [5].

Así mismo en la formación académica de aquellas personas que se están desarrollando como profesionales, esto es importante ya que pueden tomar el uso de herramientas tecnológicas como beneficio a futuro dentro de su profesión.

Por otra parte, este trabajo de investigación se lo realizó con el fin de buscar soluciones a una de las problemáticas más representativas de los últimos tiempos en el país, crear profesionales comprometidos con la mejora continua, la innovación y el compromiso de hacer cambios radicales dentro de la región, el trabajar con nuevas tecnologías le dará también a la institución una cualificación diferente frente al compromiso tecnológico con la sociedad [6].

Finalmente, la implementación de herramientas en jóvenes de esta carrera sería un paso positivo ya que se tendrían nuevas ideas para mejorar sus competencias y ser partes de la innovación dentro de las organizaciones en el país.

Una herramienta tecnológica en las carreras administrativas sirve para realizar una puesta en marcha de los aprendizajes teóricos adquiridos, de tal manera esta crea una simulación del trabajo que los profesionales se van a encontrar en las áreas laborales.

\section{III.METODOLOGÍA}

Esta investigación se categoriza como descriptiva [7], [8] y analítica [9]se desarrolla de las formas cuantitativa y cualitativa [10], por lo que se podría considerar un estudio con perspectiva mixta. Desde el punto de vista cuantitativo, se provee claridad en cuanto a sus alcances y objetivos, y con la ayuda de las herramientas de análisis: las estadísticas, causas y efectos identificados.

Como definición general, este tipo de investigación [11] representa modelos a seguir basados en técnicas específicas de observación, indagación y análisis numérico que ayudan a la interpretación de resultados, fundamentación de hipótesis, evaluación real de situaciones ya existentes y la contribución al problema de la sociedad.

Se realizó la entrevista a 1 decano y 1 director de carrera ya que al ser quienes llevan la construcción de manera general los contenidos y herramientas que revisan los estudiantes en torno a los procesos de estudio, se encuesto además a 12 docentes que son quienes llevan las cátedras de contabilidad general y contabilidad financiera de esta manera la información y datos entregados son los acordes a lo estudiado, realiza la encuesta a 60 estudiantes de la carrera de Contaduría Pública y
Auditoría con preguntas directas en torno a los conocimientos que se le entrega y a las expectativas que ellos tienen en torno a una nueva modalidad de reforzar sus conocimientos dentro de sus centros de estudios.

\section{IV.RESULTADOS}

\section{A.Entrevista a Directivos}

Una vez aplicada la entrevista a las autoridades académicas para evaluar el uso de herramientas tecnológicas las autoridades coinciden en la necesidad de actualizar las herramientas necesarias para aumentar la calidad de los profesionales que se ofrecen a una sociedad que lo necesita, si bien es cierto en la actualidad el mercado laboral es muy competitivo por el número elevado de estudiantes que ofrecen los CES, y la disminución de plazas laborales en una comunidad laboral reprimida, es por eso que los estudiantes y profesionales deben de ver en estas herramientas las oportunidades para emprender su profesión de manera independiente mientras se adquiere la experiencia necesaria para ser parte de un equipo en alguna organización.

Uno de los pilares fundamentales dentro de cada proceso educativo a nivel universitario depende también de la predisposición y compromiso que exista por parte de la comunidad educativa (Docentes, estudiantes), en la mayoría de los casos existe aquella resistencia al cambio por cierto de pequeños grupos esto nos ha llevado como líderes [12] a tomar decisiones en torno a la necesidad de unirnos a estos nuevos cambios ya que siempre el sistema educativo debe actualizarse periódicamente.

Otro de los factores que intervienen es el tradicionalismo que le damos a las carreras administrativas ya que en algunos casos la comunidad coincide en que la contabilidad debe enseñarse en libro y cuadernos para que sea mas efectiva ya que así fue como les enseñaron, si bien es cierto el método es correcto se debe dar paso al uso de estas herramientas.

Paralelamente se debe indicar que es necesario a los estudiantes enseñarles bajo el método tradicional y el nuevo método de esta forma estará preparado para tener un plan de contingencia en caso de un apagón digital o en el caso de que las organizaciones no cuenten con software contables hay que acotar en la actualidad Ecuador obliga a gran parte de las organizaciones privadas a llevar facturación electrónica lo que conlleva la obligatoriedad de tener herramientas digitales.

\section{B.Encuesta a docente.}

En la segmentación por experiencia de los docentes que cuentan con más de 10 años en la institución contamos con 8 docentes quienes indicaron que no existe la 
necesidad de cambiar la metodología en el proceso ya diseñado, ya que se ha venido trabajando de una manera efectiva y el realizar este tipo de cambios entorpece lo ya realizado, los docentes alegan que el realizar este tipo de cambios conlleva además en realizar cambios en torno a los syllabus aprobados por las autoridades respectiva, por lo que no lo ven prudente.

Lo indicado por los 4 docentes con menos de 10 años estaban de acuerdo con la propuesta de innovar los procesos en la carrera ya que la mayoría de sus estudiantes solo se dedican a estudiar y no tienen experiencia en el uso de software o ERP ya que no han trabajado, es por esto que la mayoría de profesionales tienen una menor competitividad frente a otros profesionales, por esta parte que indican que es necesario la utilización de nuevas herramientas digitales dentro del proceso de profesionalización ya que merecen una educación de calidad [13], con esto se busca la mejora de las competencias profesionales en los educando.

\section{C.Encuesta a estudiantes}

Los estudiantes analizan esta propuesta como una mejora dentro de su carrera profesionalizante ya que aumentarían sus competencias para afrontar un mercado laboral competitivo y bastante limitado, por esto analizan el aumentar el uso de tecnologías como algo positivo dentro de los procesos vinculantes, de la misma manera coinciden en la ejecución de las tecnologías como un mecanismo para generar propuestas que puedan darles un plus a sus conocimientos adquiridos, no desprestigian la labor docente pero si indican lo necesario de unirse a la era digital [14].

\section{D.Herramientas digitales en carreras contables}

La aplicación de un software dentro del proceso educativo en centros educativos de educación superior es un útil instrumento ya que se realiza la aplicación de la teoría aprendida durante su carrera profesionalizante esto dará como resultados avances significativos en el rendimiento de lo aprendido en torno a los estudiantes de Contaduría Pública y Auditoría , optimizando la obtención de los resultados de aprendizaje y ofreciendo al estudiante herramientas agiles para el procesamiento y obtención de la información contable.

Durante los primeros semestres de aplicación del software hay que considerar las limitaciones iniciales con las que cuentan los estudiantes antes de la aplicación del mismo, cabe recalcar que uno de los limitantes también definidos son los escasos recursos de los estudiantes y el poco acceso a tecnologías, solo cuentan con las herramientas prestadas por el centro de educación superior.

\section{V.CONCLUSIONES}

El uso de nuevas herramientas digitales resulta indispensable para el proceso profesional ante un país competitivo, tanto las herramientas digitales como las tradicionales son necesarias para las practicas docentes ya establecidas en la educación de tercer nivel esto contribuye significativamente a la sociedad ecuatoriana.

Para las organizaciones públicas y privadas con o sin fin de lucro necesitan profesionales capaces, eficientes que aporten a las mismas de una manera óptima y resulte parte del nuevo capital humano como se lo conoce, la promoción de actividades con el uso de TICs es necesaria para incluir cada vez más a las nuevas generaciones, teniendo que cuenta que esta es considerada como generación digital, el hecho de que este aporte con sus ideas resulta más conveniente.

Los resultados presentados por parte de quienes forman este centro de educación superior avalan la necesidad que tienen de innovar en base a las nuevas perspectivas mundiales en base a las nuevas necesidades.

\section{REFERENCIAS}

[1]G. Siemens, A learning theory for the digital age, USA, 2004.

[2]R. Nieto, «Educación virtual o virtualidad de la educación,,» Historia de la Educación, vol. 14, no 9, pp. 137 - 150, 2012.

[3]M. GARCIA, «La evaluación por competencias en la Educación Superior,» Revista de currículum y formación del profesorado, pp. 1 - 16, 2008.

[4]C. Mogollòn de Lugo, «Desarrollo de experiencias de aprendizaje virtual accesible,» EDUTEC, vol. 62, $\mathrm{n}^{\circ}$ 1, pp. 34 - 47, 2017.

[5]M. Moderos-Piñeiro, «La formación de competencias para la vida, En Ra Ximhai,» Universidad Autónoma Indígena de México, vol. 12, nº 5, pp. 129 - 144, 2016.

[6]C. Vasco, «Siete retos de la educacion colombiana para el periodo 2006 al 2019,» EAFIT, pp. 1 - 8, 2006.

[7]C. Sabino, El proceso de la Investigaciòn., Argentina: Lumen, 2013.

[8]A. Bavaresco, Proceso Metodológico en la Investigación: Cómo hacer un Diseño de Investigación, Venezuela: Imprenta Internacional C.A., 2013.

[9]J. Lopera , C. Ramìrez, M. Ucaris y J. Ortiz, El mètodo analìtico., Medellìn: Centro de Investigaciones Sociales y Humanas (CISH), Universidad de Antioquìa), 2010.

[10]C. A. Sandoval Casilimas, Investigacion Cualitativa, Bogotà: ARFO, Editores e Impresores , 2002.

[11]R. Hernandez Sampieri, C. Fernandez-Collado y P. Baptista Lucio, Metodología de la Investigación, Mexi- 
co D.F. : Mc. Graw Hill Interamericana, 2006. [12]O. Alvarado, Gestiòn educativa. Enfoques y procesos, Lima: Lima, 1999.

[13]OREAL/UNESCO, «El derecho a la educaciòn de calidadpara todos en Amèrica Latina y el Caribe,» Re-

\section{RESUMEN CURRICULAR}

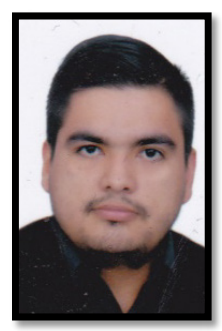

Robert Crespo López, Ecuatoriano, Ingeniero en Contaduría Pública y Auditoría de la Universidad Estatal de Milagro, Magister en Contabilidad y Auditoría de la Universidad Laica Vicente Rocafuerte de Guayaquil, maestrante del programa de Gestión educativa de la Universidad de Especialidades Espíritu Santo, docente del Ministerio de Educación del Ecuador. vista electrònica iberoamericana sobre calidad,eficacia y cambio en educaciòn, pp. 29 -35, 2007.

[14]J. Rosario, «TIC: Su uso como Herramienta para el Fortaleci,» Revistes Catalanes amb Accès Obert, 2006.

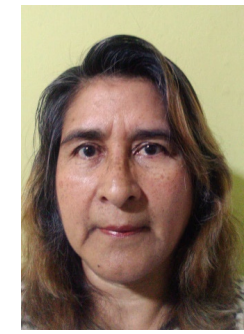

Julia López Carvajal, Ecuatoriana, Licenciada en Ciencias de la Educación con mención en Educadores de Párvulos de la Universidad de Guayaquil, Magister en Diseño Curricular de la Universidad de Guayaquil, docente del Ministerio de Educación del Ecuador. 